\title{
Purified Protein Derivative - PPD
}
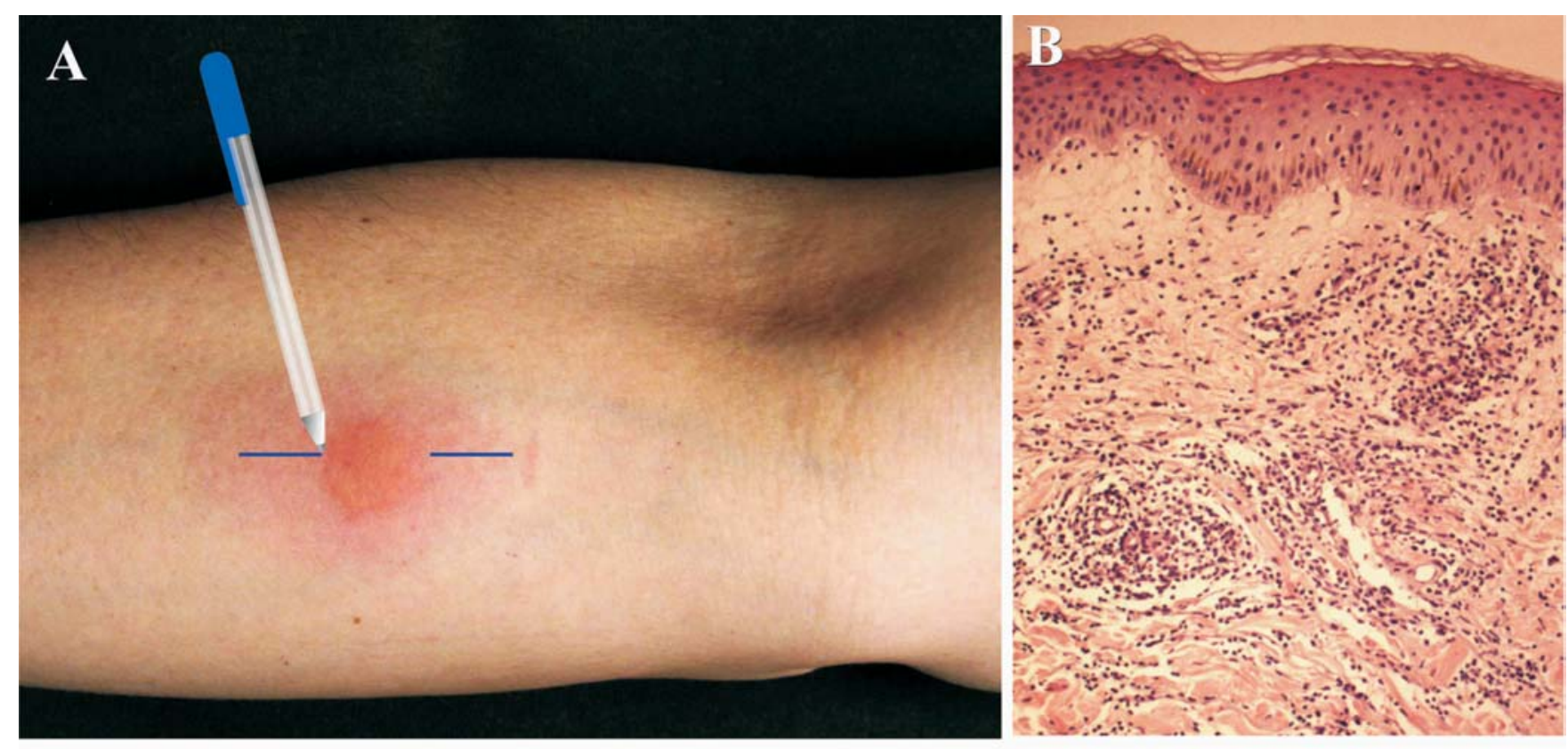

A 55-year-old physician had a PPD made as part of a clinical evaluation. Laboratory tests were unremarkable and PPD, read in 48 hours, showed an area of induration of $18 \mathrm{~mm}$ and an erythema of $51 \mathrm{~mm}$ (Panel A). A $2 \mathrm{~mm}$ punch biopsy was done in the center of the induration 96 hours after the injection. Histopathology showed an intense perivascular cuffing with macrophages and lymphocytes and edema involving the dermis. The macrophages were predominant and most of them showed cellular swelling. There was some degree of vasculitis, as shown (Panel C). A positive PPD test is generally defined as greater than $10 \mathrm{~mm}$ of (Panel B) induration, not erythema, and can be measured by using a medium-point ballpoint pen to draw a line starting 1 to $2 \mathrm{~cm}$ away

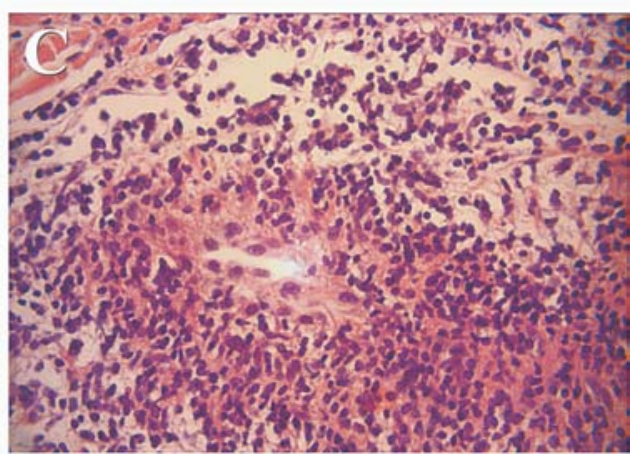

from the skin reaction (induration) and moving toward its center. The pen is lifted when resistance is felt. The procedure is repeated from the opposite direction and the distance between opposing lines is measured (Panel A). Virtually all people with PPD greater than $15 \mathrm{~mm}$ of induration are infected with Mycobacterium tuberculosis (Mtb), so the physician is infected with Mtb. PPD is also called Tuberculin and Mantoux test after the French physician Charles Mantoux (1877-1947). In 1934 Florence B. Seibert (1897-1991), an American Scientist, developed the method to obtain the Purified Protein Derivative-PPD, that became the basis for the standard Tuberculosis Test. Seibert, was a Professor of Biochemistry at the Henry Phipps Institute at the University of Pennsylvania.

Key-Words: Purified Protein Derivative, PPD, Tuberculin, Mantoux.

Anastácio Q. Sousa ${ }^{1,2}$, Margarida M.L. Pompeu ${ }^{2}$, C. Jaime Araujo Filho ${ }^{1}$, Telma R.B.S. Queiroz ${ }^{1}$, J. Clementino Ferreira ${ }^{2}$ Hospital São José and Núcleo de Medicina Tropical, Federal University of Ceará, Fortaleza, Ceará, Brazil Email:aqsousa@gmail.com

$\mathrm{C}$

The Brazilian Journal of Infectious Diseases 2006;10(6):427. () 2006 by The Brazilian Journal of Infectious Diseases and Contexto Publishing. All rights reserved. 